\title{
CONSECUENCIAS ESPACIALES DE LOS ACUERDOS DEL GATT
}

\author{
Luis SILVAN \\ Departamento de Geografía y Ordenación del Territorio \\ Universidad de Zaragoza
}

\begin{abstract}
Resumen: Un rápido análisis -desde la perspectiva geográfica- de los principales cambios que introduce y las expectativas que abre la liberalización acordada por la Ronda Uruguay del GATT: la aplicación de ella a la agricultura y los textiles, así como a algunos servicios, su repercusión en los consumidores, las diferencias de tratamiento regional, la relación comercio-medio ambiente y su consolidación institucional en la Organización Mundial del Comercio.
\end{abstract}

Palabras clave: Acuerdo General de Aranceles y Comercio, Organización Mundial del Comercio, Unión Europea, Paises en Desarrollo, Paises Menos Desarrollados, comercio exterior, multilateralismo, liberalización.

\begin{abstract}
A quick analysis -from the geographical point of view- of the main changes that introduces and the expectations that opens the liberalization that has been agreed by the GATT's Uruguay Round: its application to agriculture and textiles, as soon as to some services, its repercussion for consumers, the differences in regional treatment, the trade-environment relation and its institutional consolidation in World Trade Organization.
\end{abstract}

Key words: General Agreement on Tarifs and Trade, World Trade Organization, European Union, Developing Countries, Less Developed Countries, foreing trade, multilaterism, liberalization.

\section{EL GATT Y EL FINAL DE LA RONDA URUGUAY: LIBERALIZACION Y MUNDIALIZACION}

El 15 de diciembre de 1993 cambió el mundo. Tal vez el cambio no fuera tan espectacular como el que tuvo lugar cuando cayó el muro de Berlín, pero, por otro lado, contrariamente a ese trabajo de demolición tan necesario, el éxito de la Ronda 
Uruguay ha sido una labor de construcción... El alcance y el contenido de los acuerdos son tan vastos que quizás los árboles impidan ver el bosque ( $P$. Sutherland en su alocución al Foro Económico Mundial de Davos, enero de 1994).

El Acta Final de la Ronda Uruguay del GATT firmada en Marrakesh el 15 de abril de 1994 contiene 28 acuerdos y numerosos anexos: el mayor conjunto de medidas de liberalización del comercio de toda la historia. Los aranceles se reducen en más de un tercio, se eliminan obstáculos no arancelarios y se mejora notablemente el entorno competitivo. Para el año 2002 -final del período de aplicación- el valor del comercio mundial de mercancías experimentaría un incremento de 755.000 millones de dólares, con unas cifras de PIB mundial que GATT y OCDE cifran entre 235.000 y 275.000 millones de dólares. Se amplían y refuerzan los principios del GATT, cuya utilidad se demuestra al multiplicar por 13 el comercio mundial de mercancías en menos de medio siglo, y llevando las normas multilaterales a nuevas áreas de crecimiento: el comercio de servicios y de derechos de propiedad intelectual. La Ronda Uruguay afecta al empleo, el desarrollo de la inversión y la actividad económica toda; y su valor de conjunto se revela bastante mayor que la suma de sus partes.

Ningún país puede vivir aislado en un mundo tan interdependiente como el nuestro; ni el comercio internacional puede ser destino residual de los excedentes producidos o fuente de suministro de lo que somos incapaces de producir, pues desaprovecharíamos el mayor beneficio del intercambio: contrastar la eficacia de los procesos productivos.

En efecto, el factor que más ha influído en el crecimiento de la economía mundial en casi medio siglo ha sido la eliminación de los obstáculos comerciales, en un proceso coyunturalmente empañado por incursiones proteccionistas durante las crisis del sistema monetario, del petróleo o de la deuda exterior. Sin embargo, el propósito del GATT no ha sido instaurar la libertad en el comercio mundial, sino su liberalización dentro de una normas.

Hace años que el desarrollo económico de los países se basa -en buena medidasobre el crecimiento del comercio mundial y de las corrientes financieras, como sucede en 1992 para los países industriales. Por tanto el fortalecimiento de las normas multilaterales y la liberalización del comercio en todos los sectores, contribuirá al desarrollo de la actividad económica global durante los próximos años.

Las negociaciones emprendidas en 1986 por la Ronda Uruguay pretendían consolidar un sistema de comercio internacional, libre y no discriminatorio, mejorar sus normas y mecanismos, así como introducir la disciplina del GATT en los sectores no industriales. Prevista inicialmente para cinco años, su complejo contenido, la 
dificultad de armonizar los intereses comerciales estatales y regionales, así como la coyuntura económica internacional la han prorrogado dos años más.

Por nuestra parte destacaremos aquí y ahora algunas repercusiones geográficas de los acuerdos de esta octava Ronda del GATT, que por primera vez incluye la agricultura y los textiles, así como algunos servicios; cómo afecta la liberalización a los consumidores, las medidas de tratamiento regional, la relación comercio-medio ambiente y su consolidación institucional en la Organización Mundial del Comercio.

\section{ASPECTOS SECTORIALES: LA AGRICULTURA Y LOS SRVICIOS}

Los productos agrarios habían sido excluídos tradicionalmente del GATT por la variabilidad de su producción y su general protección a nivel mundial; de ahí la trascendencia y complejidad del Acuerdo sobre la Agricultura conseguido en la Ronda Uruguay, que supone un avance importante sobre sus objetivos iniciales:

- mejorar el acceso al mercado, reduciendo los obstáculos a las importaciones,

- mejorar la competencia, disciplinando las subvenciones y otras medidas, y

- armonizar las reglamentaciones fitosanitarias y veterinarias.

El reforzamiento de las normas del comercio de productos agropecuarios mejora las condiciones de previsión y estabilidad para los países importadores y exportadores de los mismos. El conjunto de disposiciones se concibe como parte de un proceso contínuo con el objetivo a largo plazo de reducir substancial y progresivamente la ayuda y la protección de la agricultura.

Cuatro partes presenta el referido Acuerdo:

- el Acuerdo sobre la Agricultura propiamente dicha plantea un marco para la reforma a largo plazo del comercio de los productos y de las políticas agropecuarias internas, con la finalidad de orientarlos al comercio mediante el reforzamiento normativo. Para las políticas de ayuda interna prevé medidas sostenedoras de la economía rural sin distorsionar el mercado; así como toma en consideración a los países en desarrollo, en particular los países importadores netos de alimentos y los menos desarrollados.

- En la esfera del acceso a los mercados, reemplaza las medidas no arancelarias en frontera por aranceles de un nivel de protección equivalente. Estos y demás 
aranceles agropecuarios han de reducirse un $36 \%$ de promedio en los países desarrollados (PD) y un $24 \%$ en los países en desarrollo (PED), a lo largo de seis o diez años; no exigiéndose tal reducción a los países menos desarrollados (PMD). Para facilitar la aplicación de la arancelización en situaciones particularmente sensibles se introduce una cláusula de trato especial para ciertos países, que permite mantener las restricciones a la importación hasta finalizar el período de aplicación.

- Con respecto a la Medida Global de Ayuda (MGA) a la agricultura, y que comprende toda la ayuda -no exenta- recibida por productos específicos, la Ronda Uruguay se ha comprometido a reducirla un $20 \%$ (13,3\% para los PED y nada para los PMD), a lo largo del período de aplicación. Las medidas de ayuda interna del compartimento verde -o de impacto mínimo- están excluídas de la reducción: es decir, los servicios generales del gobierno, la asistencia para el reajuste estructural, los pagos directos en el marco de los programas ambientales y de asistencia regional. La CE ha conseguido también esa calificación en los pagos directos a los agricultores para sostener sus ingresos al margen de la producción. Tampoco se incluyen los pagos directos en programas de limitación de la producción, ciertas medidas oficiales de asistencia para fomentar el desarrollo agrícola y rural de los PED y otras ayudas que representen solamente una proporción reducida del valor de producción específica o total.

- Por último, se requiere que los países miembros del GATT reduzcan -entre 1995 y el 2000 - el $33 \%$ en valor de las restituciones a la exportación respecto al período base 1986-90; así como el $21 \%$ en cantidad de las exportaciones subvencionadas; reducciones que para los PED suponen las $2 / 3$ de las de los PD y a lo largo de diez años.

El Acuerdo sobre los Textiles y el Vestido consigue por fin sujetar este importante subsector del comercio mundial a las normas y disciplinas reforzadas del GATT, a lo largo de un período dilatado hasta el año 2005.

Por su parte, el Acuerdo General sobre el Comercio de Servicios contiene tres elementos principales: un acuerdo marco con las obligaciones básicas aplicables a todos los países miembros, listas nacionales de compromisos y varios anexos relativos a la especialidad de los distintos subsectores de servicios.

Entre las obligaciones básicas contenidas en el marco de normas y disciplinas generales destaquemos la cobertura global, que depende del tipo de servicio, que puede ser suministrado de un espacio a otro, en un espacio a los consumidores de otro (turismo), por entidades proveedoras de servicios de un espacio en el de otro (servicios bancarios), o por nacionales de uno en otro (proyectos de construcción o servicios de consultoría). Además del trato nacional, trato de nación más favorecida, transparencia y reconocimiento. 
El anexo sobre movimiento de personas físicas permite a los gobiernos negociar compromisos específicos sobre la estancia temporal de personas con el fin de prestar un servicio, pero sin ser de aplicación a aquéllas que traten de obtener empleo o residencia con carácter permanente en un país.

El anexo sobre servicios financieros afecta principalmente a los servicios bancarios y de seguros, con posible adopción de medidas cautelares.

El anexo sobre telecomunicaciones reconoce su doble función de sector independiente de actividad económica y medio fundamental de transporte de otras actividades. Fomenta la cooperación técnica para ayudar a los PED a fortalecer sus propios sectores nacionales de telecomunicaciones.

El anexo sobre los servicios de transporte aéreo excluye del ámbito de aplicación del acuerdo los derechos de tráfico y actividades directamente relacionadas con éstos. Sin embargo, se aplicará a servicios de reparación, mantenimiento de aeronaves, comercialización de servicios de transporte aéreo y servicios de reserva informatizados.

Resultado de las negociaciones bilaterales, cada gobierno consigna en su lista nacional sus compromisos iniciales de acceso a los mercados y las limitaciones que desea mantener respecto a los principios básicos de la cobertura global de los grandes sectores de los servicios, y al trato nacional.

\section{ASPECTOS POBLACIONALES: LOS CONSUMIDORES}

Más que en la producción de bienes o servicios, todos nos vemos de alguna manera implicados en su consumo. El control de las ayudas a la actividad productiva y la liberalización de los intercambios suponen beneficios para el consumo intermedio o final. Por eso el interés de los consumidores ha sido esgrimido en las negociaciones de la Ronda Uruguay -sobre todo desde sus últimas prórrogas- y presentado ante la opinión pública como sólido argumento para inclinar la balanza decisoria frente a los intereses específicos de los poderosos productores nacionales. Pues en la práctica todo tipo de protección origina precios más elevados, que alguien habrá de pagar: otro productor en el caso de los bienes intermedios, o el consumidor final donde nos incluimos.

Su consecuencia es un descenso de los ingresos reales y privarnos de otros bienes o servicios, aunque en un mundo social, informado y consciente de sus derechos y bienestar, el consumidor no debiera pagar cargas innecesarias y costes excesivos. Pero 
la realidad es que muchas de las medidas que adoptan los gobiernos para proteger aparentemente los productos nacionales frente a los extranjeros -aranceles y restricciones cuantitativas a la importación, limitaciones voluntarias de exportación, subvenciones a la producción, derechos antidumping y compensatorios, obstáculos técnicos al comercio-, termina por pagarlas directa o indirectamente el propio consumidor. Un producto gravado con un arancel del $20-30 \%$ incrementa su precio en tienda más que el gravámen aduanero. Los contingentes y las restricciones voluntarias -limitando el acceso de bienes al mercado- no sólo propician el incremento de los precios internos, sino cierto desabastecimiento. Mencionemos los ejemplos ya clásicos:

- los automóviles, donde Estados Unidos y la Comunidad Europea se protegen desde hace tiempo de sus competidores japoneses mediante contingentes de importación o limitaciones voluntarias de exportación, esas restricciones costaron en 1993 a los consumidores de cinco países comunitarios unos 7.000 millones de dólares;

- gracias al Acuerdo Multifibras los textiles y el vestido de los países industriales se han defendido de las exportaciones del Tercer Mundo, calculándose el coste de la protección sólo para Estados Unidos en unos 27.000 millones de dólares ;

- el Japón no deja entrar en su mercado un grano de arroz desde 1967, gracias a la cual cuesta allí 4-5 veces más que en Estados Unidos; en fin,

- las transferencias totales -en aumentos de precios e impuestos- de los consumidores a los productores para pagar la ayuda oficial a la agricultura en los países de la OCDE ascendieron en 1992 a 3537 miles de millones de dólares, es decir 440 dólares por habitante (vid. Tabla 1 ).

La situación resulta particularmente gravosa para los sectores sociales más pobres, que gastan la mayor parte de su presupuesto en artículos de primera necesidad -alimentación y ropa- donde la protección es más frecuente e intensa. Los consumidores de los países en desarrollo también se ven perjudicados por la protección de las importaciones. Además de incrementarse el coste de los productos, otras secuelas frecuentes en el mercado suelen ser la menor variedad de bienes o la penuria del abastecimiento. Las consecuencias se acusan además de las unidades familiares, en aquellas industrias que consumen productos de importación, cuya competitividad interior y exportadora depende de la obtención de productos buenos y baratos. 
Tabla 1.- Transferencias de los consumidores a los productores para pagar la ayuda oficial a la agricultura.

\begin{tabular}{|l|c|c|}
\hline \multicolumn{1}{|c|}{ PAIS } & $\begin{array}{c}\text { Totales en miles de } \\
\text { millones \$ EE.UU. }\end{array}$ & $\begin{array}{c}\text { \$ EE.UU. } \\
\text { por habitante }\end{array}$ \\
\hline Australia & 1.6 & 89 \\
\hline Austria & 4.2 & 530 \\
\hline Canadá & 9.1 & 330 \\
\hline CE & 155.9 & 450 \\
\hline Finlandia & 4.5 & 910 \\
\hline Japón & 74.0 & 600 \\
\hline Nueva Zelanda & 0.1 & 15 \\
\hline Noruega & 4.1 & 970 \\
\hline Suecia & 3.2 & 370 \\
\hline Suiza & 5.8 & 840 \\
\hline Estados Unidos & 91.1 & 360 \\
\hline Total OCDE & 353.7 & 440 \\
\hline
\end{tabular}

El argumento habitualmente aducido para las medidas protectoras es el mantenimiento del empleo interior, y podía servir de ser verdad; pero con frecuencia el coste de salvar un puesto de trabajo es superior al sueldo que supone, por lo cual sería más útil destinarlo a programas de reconversión, con ayudas a la capacitación profesional y la movilidad de la población activa.

\section{ASPECTOS REGIONALES: PAISES DESARROLlADOS, EN VIAS DE DESARROLLO Y MENOS DESARROLLADOS}

Desde el inicio de la Ronda Uruguay en 1986 el GATT ha aumentado de 89 a 126 sus miembros, demostrando así que todos los países ganan con la apertura y liberalización del comercio, sean desarrollados, en desarrollo o menos desarrollados. El GATT pasa de club relativamente pequeño y cerrado, a institución de amplia representación geográfica, cuyas actividades son objeto de debate por todo el mundo.

Para cualquier país partícipe de la Ronda Uruguay, el aumento del PIB derivado de su feliz conclusión tendrá como fuentes principales: 
- un uso más eficiente de los recursos internos con la supresión de sus barreras comerciales, y

- un mayor acceso a los mercados de los otros países.

Los acuerdos adoptados permitirán a todos los países del mundo incrementar sus exportaciones, requisito imprescindible para poder mantener o aumentar las importaciones, atraer capitales y mejorar las condiciones económicas y sociales de sus respectivos espacios. La valoración de sus consecuencias en los diversos regiones debe realizarse sobre una triple premisa:

- el orden y previsión que se derivan de unas relaciones comerciales basadas en normas, y no sólo en el peso económico;

- el efecto dinamizador de la conclusión de la Ronda sobre los círculos mercantiles y empresariales, y

- la consideración de los resultados de la Ronda como un conjunto, en el que todos pierden y ganan.

Pero igual que la Ronda no habría concluído satisfactoriamente sin sus diversos acuerdos -de la agricultura, servicios, etc-- tampoco lo habría hecho si hubiesen desertado grupos de países o los más significativos: su mayor valor es la apreciación global o de conjunto. Entre los PD o de la OCDE se han buscado buenos y malos; pero si confrontan los intereses de unos $u$ otros países ante el GATT, es por su diferente participación en el comercio mundial, del comercio en el PIB, de su destino regional o exterior, de la naturaleza o composición de los intercambios, del grado liberalizador de que se parta, etc., que difieren entre los países de la Unión Europea, Estados Unidos, Japón u otros del grupo de Cairns, como demuestran su diferente crecimiento anual del comercio o PIB.

El dinamismo económico y comercial de la OCDE hace de motor impulsor de los intercambios con los PED y PMD, y todos los países en general se aprestan al juego del multilateralismo: los PD que sufren las consecuencias de la desaceleración del crecimiento y el desempleo, los países en transición que se abren costosamente al mercado, los países agrícolas eficientes que ven amenazadas sus exportaciones y los PED que intentan renegociar su deuda. Pero mejorando las oportunidades de exportación de los PED, que incluyen las economías de más rápido crecimiento del momento (los dragones asiáticos, América Latina y Europa Central y Oriental), también se mejoran las expectativas de los países industrializados de convertir su frágil recuperación actual en un crecimiento sostenible. 
Desde su comienzo, la Ronda se ha preocupado de adaptar el programa de reforma a los diferentes espacios regionales, ya que los PMD y los PED importadores netos de alimentos pueden experimentar efectos negativos de la liberalización: sus Acuerdos promueven medidas positivas, exigencias menores o exenciones en la cuantía y calendario de liberalización arancelaria (vid. supra), incluso con suministro de ayuda alimentaria y de productos alimenticios básicos en forma de donación completa y ayuda para el desarrollo agropecuario.

Los críticos aducen que éstos países obtendrán una doble pérdida económica con las innovaciones de la Ronda Uruguay: la primera, relacionada con la importación de productos alimenticios, cuyo precio se calcula aumente un $5 \%$ en los próximos años; pero se contrarrestará con la gradualidad de la reforma, mayor estabilidad de precios y posibilidad de modificar la producción. La segunda es la reducción de los márgenes de preferencia de que actualmente disfrutan las exportaciones enviadas de los PED a los $\mathrm{PD}$, que se compensa con una mayor certeza en la reducción del trato de nación más favorecida, diversificando sus destinos geográficos, o con su transformación productiva.

\section{ASPECTOS MEDIOAMBIENTALES}

El proceso de mundialización de las actividades geográficas coincide con el descubrimiento y la concienciación acerca de los problemas medioambientales. Sólo que mientras las primeras suelen ser inducidas desigualmente por los distintos países, los segundos son padecidos con independencia de su autoría.

Es opinión bastante arraigada que la conclusión de la Ronda Uruguay favorece per se el desarrollo sostenible. En efecto, una mayor liberalización del comercio, con un acceso más amplio a los mercados asignaría más eficientemente los recursos nacionales, reduciendo al mínimo los desechos y la contaminación; mientras que la disminución de los obstáculos a las exportaciones de los PED fomentaría el crecimiento económico y generaría más recursos para elevar la calidad del medio ambiente a nivel nacional.

Igual que la política ambiental tiene sus aspectos económicos, el comercio origina problemas medioambientales; por lo cual es lógico plantear una economia política de las interacciones entre políticas ambientales y comerciales. En los últimos años se asume la idea de que ciertas manifestaciones cuantitativas, compositivas y distributivas del comercio internacional están produciendo consecuencias espaciales indeseables sobre el medio ambiente. 
Aunque hace ya tiempo que se observan las implicaciones entre desarrollo y medio ambiente, su compleja problemática explica la parquedad y lentitud de los avances. El Acuerdo que establece la OMC hace suyos los objetivos del GATT, entre ellos utilizar de forma óptima los recursos mundiales, y -precisándolos- introduce el principio del desarrollo sostenible.

En este tema -más aún que en otros- los países discrepan. Brasil es firme partidario de incorporar al GATT los principios del Programa 21 de la CNUMAD, ya que la pobreza es el peor contaminante en el mundo en desarrollo. El espíritu de Río se infunde -pues- en la organización comercial naciente, tratando de compatibilizar un sistema multilateral de comercio abierto, no discriminatorio y equitativo, con las medidas de protección del medio ambiente y la promoción de un desarrollo sostenible; para lo cual habrá que coordinar las políticas comercial y medioambiental en el ámbito multilateral de comercio.

La decisión adoptada en Marrakesh sobre comercio y medio ambiente compromete la creación de un Comité, cuya competencia especial será la relación entre las disposiciones del sistema multilateral de comercio y las medidas comerciales adoptadas con fines ambientales, y viceversa; también en la exportación de mercancías cuya venta está prohibida en el país de origen, y sobre el efecto de las medidas ambientales en el acceso a los mercados y -a la inversa- sobre los beneficios medioambientales de la eliminación de restricciones y distorsiones comerciales.

\section{ASPECTOS INSTITUCIONALES: LA ORGANIZACIÓN MUNDIAL DEL COMERCIO}

La Organización Internacional del Comercio va a ser desde 1995 la institución sucesora del GATT, un Acuerdo formal que ocupó provisionalmente el tercer pilar económico -o comercial- de la postguerra en lugar de la non-nata Organización Internacional del Comercio. Serán sus objetivos:

- facilitar la puesta en práctica y aplicación de todos los Acuerdos e instrumentos jurídicos negociados en el marco de la Ronda Uruguay;

- servir de foro a todas las negociaciones comerciales;

- administrar el Entendimiento sobre Solución de Diferencias y el Mecanismo de Examen de las Políticas Comerciales; 
-cooperar con el FMI y el BIRD para lograr una mayor coherencia en la elaboración de las políticas económicas a escala mundial.

El Acuerdo que constituye la OMC asume los objetivos del GATT: elevar los niveles de vida y de renta, lograr el pleno empleo, acrecentar la producción y el comercio y utilizar de forma óptima los recursos mundiales, extendiéndolos al sector de los servicios, y precisándolos, establece la idea del desarrollo sostenible con respecto a la utilización óptima de los recursos mundiales y la necesidad de proteger y preservar el medio ambiente de manera compatible con los distintos niveles de desarrollo económico de los países; reconociéndose que es necesario realizar esfuerzos positivos para que los PED y sobre todo los PMD, participen más en el incremento del comercio mundial.

La idea de un nuevo orden mundial del comercio es vital para todos los países, especialmente par los pequeños y pobres. A todos beneficiará el acceso a los mercados, sometido a unas reglas de juego. La Ronda Uruguay proyecta su dinamismo en la OMC, que no sólo deberá aplicar sus Acuerdos, sino que será el foro de nuevas negociaciones comerciales, del creciente intercambio de servicios y de las nuevas cuestiones planteadas por el comercio mundial: su vinculación con el medio ambiente, la política en materia de competencia internacional, el comercio y la inversión, y el comercio y las normas laborales.

\section{CONCLUSIONES}

La Ronda Uruguay constituye un hito importante, todo un récord entre las Rondas de negociación comercial y su más plena planetarización normativa, de la que son protagonistas un centenar amplio de partes contratantes del GATT. De esta forma las relaciones comerciales multilaterales se entrecruzan por el espacio planetario, afectando la actividad económica toda, arrumbando el proteccionismo, mejorando el comercio bilateral y potenciando el desarrollo socioeconómico de todas las regiones del espacio-mundo.

El proceso iniciado en Marrakesh con la firma de los Acuerdos de tan ardua negociación comercial ha de cerrarse ya con la ratificación preceptiva por parte de todos y sus más significativos firmantes. Sólo este impulso final permitirá a la OMC alumbrar -a partir de enero de 1995- una nueva era de relaciones comerciales pacíficas. Ambos eventos institucionales contienen un mensaje de esperanza en la capacidad de la humanidad para avanzar hacia la consecución de un mundo económico más justo. 


\section{BIBLIOGRAFIA}

Acuerdo General sobre Aranceles Aduaneros y Comercio (1991): GATT, ce qu'il est, ce qu'il fait. GATT,. 45 p. Ginebra.

Acuerdo General sobre Aranceles Aduaneros y Comercio (1993): El Comercio Internacional 1993. Estadísticas. GATT, 120 p. Ginebra.

Acuerdo General sobre Aranceles Aduaneros y Comercio: Focus, Boletín de información. GATT, Ginebra.

ANDERSON, K. y BLACKHURST, R. Dirs. (1992): El comercio mundial y el medio ambiente. Mundi-Prensa, 300 p. Madrid.

BRIZ, J. y MARTIN, M. (1991): Politicas comerciales internacionales agrarias. E. Agrícola. Madrid.

CASTILLO, J.A. (1991): "Esbozo de una explicación de la Ronda Uruguay". Revista de Estudios Agro-Sociales, núm. 155. 79-103. Madrid.

DEZERT, B. et WACKERMANN, G. (1991): La nouvelle Organisation des échanges. Dossiers des Images économiques du monde. Sedes. 234 p. Paris.

FELIPE, I. DE (1991): "La Política Agraria Común, el GATT y los Países en Vías de Desarrollo" El Campo, núm. 122. Fundación BBV. 62-65, Bilbao

GARCIA ALVAREZ COQUE, J.M. (1991): "Las propuestas de liberalización del comercio mundial agropecuario. Una aproximación cualitativa". Revista de Estudios Agro-Sociales, núm. 155. 11-39, Madrid.

GOMEZ TORAN, P. (1991): "Políticas de ayuda y protección a la agricultura: su tratamiento en el GATT". Revista de Estudios Agro-Sociales, núm. 155, 105-129, Madrid.

JARA., A. (1990): "La Ronda Uruguay del GATT y los Países en Desarrollo". Revista de Estudios Agro-Sociales, núm. 152. 93-100, Madrid.

LOPEZ CORDOVEZ, L. (1991): "Perspectivas del comercio mundial agroalimentario". Revista de Estudios Agro-Sociales, núm. 152. 43-46, Madrid.

MARTIN, M.M. (1991): "La agricultura da Estados Unidos frente a la europea en la liberalización del comercio agrario". Revista de Estudios Agro-Sociales, núm. 155. 55-67, Madrid.

MILAN DIEZ, R. (1994): "El Acuerdo Final sobre Agricultura en la Ronda Uruguay". El Boletín, núm. 12. M.A.P.A., 6-17, Madrid. 
OCDE: Statistics of Foreing Trade.

SAN JUAN MESONADA, C. (1991):"La Ronda Uruguay del GATT. La dimensión internacional". Revista de Estudios Agro-Sociales, núm. 155. 193-197, Madrid. 